\title{
Minimal Cut Sets-Based Reliability Evaluation of the More Electric Aircraft Power System
}

\author{
Yuancheng Zhao $\mathbb{D}^{1},{ }^{1}$ Yanbo Che $\mathbb{D}^{1},{ }^{1}$ Tingjun Lin, ${ }^{2}$ Chuanyan Wang, ${ }^{3}$ Jiaxuan Liu, ${ }^{4}$ \\ Jianmei Xu $\left({ }^{\circ},{ }^{1}\right.$ and Jinhuan Zhou $\left(\mathbb{1}^{1}\right.$ \\ ${ }^{1}$ Key Laboratory of Smart Grid of Ministry of Education, Tianjin University, Tianjin 300072, China \\ ${ }^{2}$ College of Energy and Electrical Engineering, Hohai University, Nanjing, Jiangsu Province 210098, China \\ ${ }^{3}$ Shandong Light Industry Engineering College, Qingdao, Shandong Province 266112, China \\ ${ }^{4}$ College of Information and Control Engineering, China University of Petroleum, Qingdao, Shandong Province 266580, China
}

Correspondence should be addressed to Yanbo Che; ybche@tju.edu.cn

Received 12 July 2017; Revised 4 December 2017; Accepted 25 December 2017; Published 28 February 2018

Academic Editor: Ivan Giorgio

Copyright (c) 2018 Yuancheng Zhao et al. This is an open access article distributed under the Creative Commons Attribution License, which permits unrestricted use, distribution, and reproduction in any medium, provided the original work is properly cited.

\begin{abstract}
The More Electric Aircraft (MEA) stands for the direction of aviation development in the new era, and the reliability of power systems on the MEA has attracted widespread attention. Based on the characteristics of MEA power systems, an equivalent method of electrical topology structure is presented in this article, and evaluation method is proposed which shows the reliability of the overall system with the reliability of specific nodes. Firstly, electrical topology structure of a MEA power system is converted into a network node diagram according to the proposed equivalent method. Then, the minimal path sets of specific nodes are obtained by the adjacent matrix algorithm, and the low-order minimal cut sets of disjointed are obtained. After that, the actual failure rate of components is converted to node failure rate, and the reliability of the overall system is evaluated by operational reliability indexes of specific nodes. Finally, taking the MEA A380 as an example, this paper compares and analyzes the reliability of AC loads, DC loads, and key loads to verify the validity and feasibility of the proposed evaluation method. This evaluation system can predict the weak points existing in the MEA power system, as well as providing theoretical support for maintenance schedule.
\end{abstract}

\section{Introduction}

MEA is one kind of aircraft that replaces the original hydraulic, pneumatic, and mechanical drive systems with power systems as many as possible [1]. The power system studied in this paper is one of core systems in MEA, which is the general term of power generation, transmission, conversion, and consumption on an aircraft [2]. Compared with traditional aircraft, the MEA has advantages of simple structure, light weight, high efficiency, easy maintenance, low fuel consumption, and strong vitality $[3,4]$. Therefore, the research and application of MEA have received great attention both at home and abroad in recent years.

The development of MEA power system plays a vital role in multiaircraft electrification and even full. The extremely high reliability of aircraft operation has prompted reliability of MEA power system to be a hot research topic in the field of aircraft safety [5]. A MEA power system has the characteristics of complex structure, redundant lines, many power electronic devices, and so on which pose new requirements and challenges for the reliability analysis of the aircraft power system.

Up to now, no systematic research has been found on the reliability evaluation of EMA power system. A MEA power system during operation can be regarded as an irreparable microgrid but different from an ordinary microgrid for its requirement of high reliability and characteristics of operation. Only for the aircraft in operation the assessment of reliability is of value, so this article does not consider the external power supply. Therefore, the reliability evaluation methods for traditional power grid have strong reference value. Reference [4] proposes a topological method for quantitative analysis of the power system of MEA, and Euclidean distance and entropy weight are used to evaluate the analysis results. 
In the literature [6], the analytical technique combined with the average recovery time is compared with the Monte Carlo method combined with random restoration time. Reference [7] presents a probabilistic technique to assess quantitatively the reliability impact of wind-based distribution generations in the microgrid during islanded mode of operation. In the literature [8], a new approach is used to implement the optimization of reliability with the help of Fault Tree Analysis in the grid-connected microgrid.

Most of these literatures studied the power system as a repairable system, which is not applicable to MEA power system. Only by improving reliability of components, optimizing structure, and setting up multiple redundancies can an irreparable system enhance its anti-interference ability and self-healing capability, which is different from reparable system. For an irreparable system, any failure is often fatal, so reliability evaluation is vital of importance. The State Space Method and Monto-Carlo Simulation generally effective in reparable systems are no longer applicable in irreparable systems; hence, it is required to propose a new and targeted equivalent method and evaluation method according to the characteristics.

Based on theory of graph, this paper presents the equivalent method for characteristics of EMA power system, by which complex electrical topology structures are converted into network node diagrams. Based on the minimal cut sets, a reliability evaluation system is established. Under the condition of the known fault data and system structure, its purpose is to describe the reliability of the overall system with the reliability indexes [9], such as the probability of some key components failure.

The evaluation system processes mainly include the following: the minimal path sets are obtained from the source node to specified nodes by the adjacent matrix algorithm; low-order minimal cut sets of disjointed are calculated; the actual failure rates of components are converted into the corresponding node failure rate. Reliability of the overall system is evaluated by reliable operation probability of specific nodes-node failure rate. Finally, MEA A380 is taken as an example to validate the feasibility and effectiveness of the proposed method.

\section{A Brief Introduction of the MEA Power System}

MEA is the first step for the full electrification of aircraft, and this change takes a long time. At this stage, only three kinds of aircraft (A380, B787, and F-35) can be called real MEA [10]. Next is a brief introduction to the composition, operation, and characteristics of the power system, and A380 is taken as an example. Figure 1 shows the electrical topology structure of A380.

The main power supply of A380 includes 4 variable frequency AC generators, of which capacity is $150 \mathrm{kV} \cdot \mathrm{A}$, regulating voltage is $115 / 200 \mathrm{~V}$, and operating frequency range is $370 \sim 770 \mathrm{~Hz}$. Meanwhile, there are 2 constant frequency $400 \mathrm{~Hz}$ generators as Auxiliary Power Unit (APU) with and capacity of $120 \mathrm{kV} \cdot \mathrm{A}$, and there are 4 types of external power access for ground power supply. The emergency power supply of A380 includes $70 \mathrm{kV} \cdot \mathrm{A}$ Ram Air Turbine (RAT) and battery with capacity of $50 \mathrm{~A} \cdot \mathrm{h}$.

The A380 has four separate main AC bus bars that accept power from ground supply and main generators, respectively. The AC bus bars could not operate in parallel, because the variable frequency of main generators is related to speed of turbine engines. Four AC bus bars are converted into DC bus bars through power electronic converters. The DC system has the ability to provide uninterrupted power; thus it ensures uninterrupted power supply for key loads when the topology changes.

As shown in Figure 1, the overall power system is roughly divided into 4 main channels: E1 channel supplied by AC generators G1 and G2; E2 channel supplied by AC generators G3 and G4; E3 channel supplied by RAT and static converters; and APU channel associated with APU startup.

The layout combines the advantages of both the parallel operation and the independent operation. The DC system in parallel ensures uninterrupted power supply and keeps the system stable by buffering the transient changes due to load fluctuation and operation mode conversion. The independent operation of channels can effectively prevent cascading failures. If the faults occur independently, the fault can be blocked in a channel without affecting the entire system.

In summary, MEA power system has characteristics of high redundancy, complex structure, and more various power electronics.

\section{Equivalent Method of Electrical Topology Structures}

It is necessary to make the electrical topology structures equal to network node diagrams for computer assisted calculation and analysis. Combined with the theoretical basis of graph theory, simple network node diagrams are used to show logical relationship of electrical topology structure. In order to fully highlight the characteristics of electrical topology structure in MEA, an applicable equivalent method is presented.

Assume that each component has only two states of work or failure. Isolating switches and channel switches are controlled by actuators, and they could turn on or off automatically on the demand of reconfiguring system topology. The below principles of equivalence are followed, when the electrical topology structures are converted into network node diagrams: the network node diagrams are able to reflect accurately the connection relationship and working state of the original topology; the network node diagrams should be as simple and convenient as possible to make full use of computer assistance.

Details are as follows.

Components with equal voltage roughly can be equivalent to nodes, such as bus bars and distribution devices. Components with obvious voltage difference can be equivalent to arcs, such as impedance and generators converter transformers. The power channels where components control the flow of energy are abstracted as directed arcs, otherwise, undirected arcs. The essence of undirected arc is that energy 


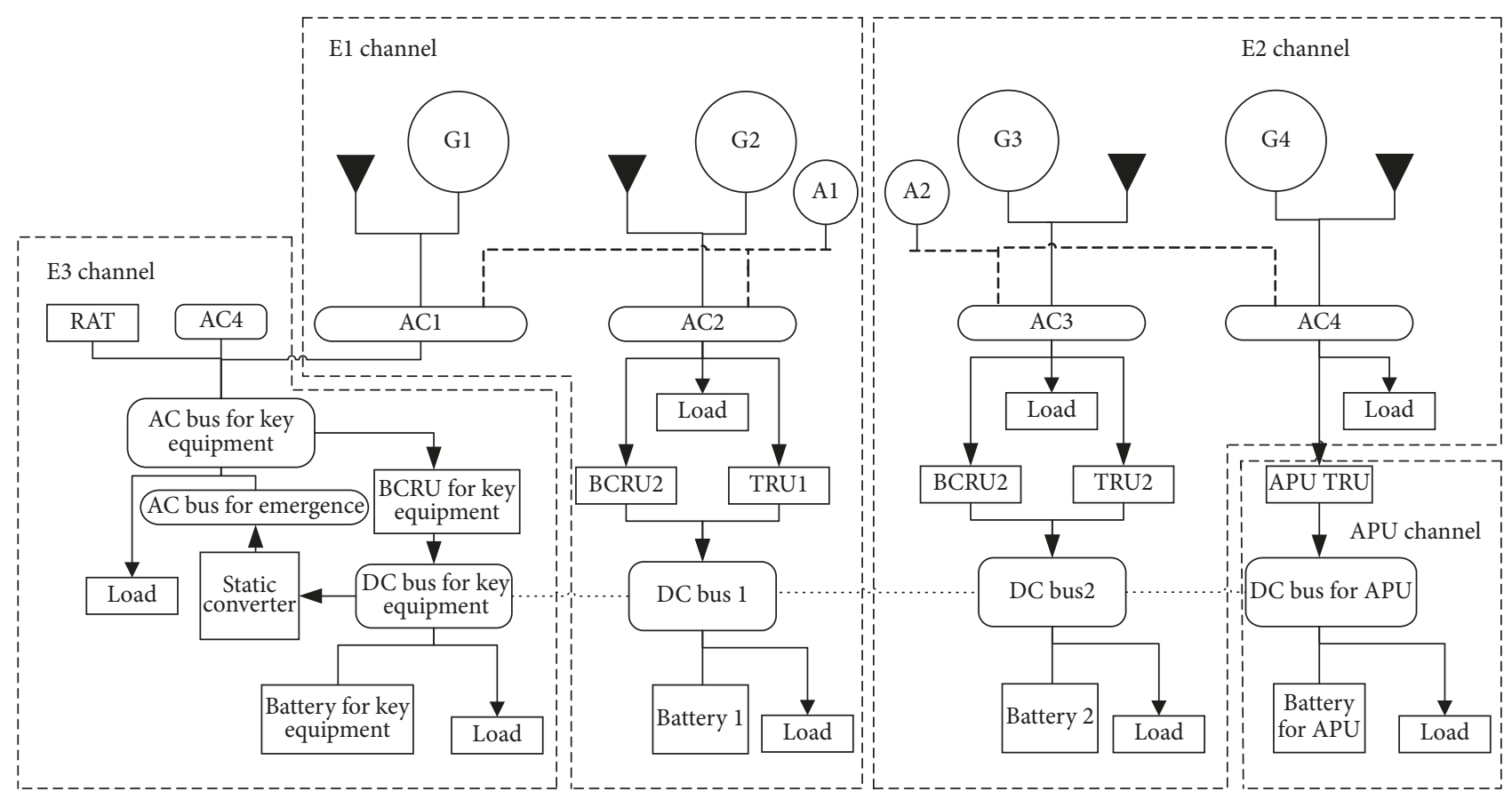

FIGURE 1: Electrical topology of A380.

flows bidirectionally, so the undirected arc is equivalent to bidirectional arc.

Set up a virtual source node as the power input of the entire microgrid. Some trap nodes are set to stand for loads. Trap nodes and arcs both can consume energy.

To reduce the number of nodes and arcs in the diagram, several arcs are allowed to be equivalent to one single arc when elements are connected in series without branch. The trap nodes representing loads are allowed to been absorbed by directed arcs. Figure 2 is obtained after the change of Figure 1, based on the above equivalent method.

Comparing Figures 1 and 2, the power of four bus bars $\mathrm{AC1}(\mathrm{v} 2), \mathrm{AC} 2(\mathrm{v} 4), \mathrm{AC} 3(\mathrm{v} 7)$, and $\mathrm{AC} 4(\mathrm{v} 9)$ is supplied from the virtual source Node $\mathrm{vl}$, through four generators equivalent to arcs. Arc e21 expresses the fact that power of AC bus bars for key equipment is generated from RAT to keep them working properly on emergency. Nodes v3, v5, v7, and v9 are trap nodes representing AC loads, while Nodes v16, v17, v18, and v19 are trap nodes representing DC loads, and Node v20 stands for the key load that maintains flying operation. In particular cases, the battery can supply power for Node v1l representing bus bars reversely via Nodes v12, v13, v14, and v15. For convenient calculation, the three batteries are absorbed into the nodes of direct connection that is the corresponding DC bus bars. It expresses the state of parallel operation of the DC system that undirected arc is connecting four Nodes v12, v13, v14, and v15. Normally, there is no energy flow on the parallel channels. DC bus bars with no power are supplied by DC bus bars with power after topology reconfiguration. For reduction of nodes, undirected arcs are used to express normal state and reconstruction after faults without affecting the evaluation standard. Node v10 has two functions; on one hand, it can express that power from APU goes into AC bus bars; on the other hand, it can express state of AC channels operating independently.

\section{Evaluation Method of Reliability}

For evaluating the reliability of the entire MEA power system, typical nodes of power supplies and key loads are found according to characteristics of MEA power supply. Based on the minimal cut sets causing power interruption, the reliability indexes are calculated to show reliability of the entire system. In the case of failure of power topology reconfiguration, the standard of system failure is that interruption of key loads which is also regarded as the reliability evaluation standard. The overall evaluation method is shown in Figure 3.

4.1. Calculation of Minimal Path Sets. Adjacent matrix method is used to calculate minimal path sets from the source node to specific nodes, which is introduced as follows. Suppose a network nodes diagram $N=(V, E)$, where $V=$ $\left\{v_{1}, v_{2}, v_{3}, \ldots, v_{n}\right\}$ is a set of nodes and $E=\left\{e_{1}, e_{2}, e_{3}, \ldots, e_{n}\right\}$ is a set of arcs.

The electrical distance between the two interconnected nodes is defined as 1 . Adjacent matrix $A_{1}=\left[a^{1}{ }_{i j}\right]$ describes the topology information of a network.

$$
a_{i j}^{1}= \begin{cases}v_{i} v_{j} & \text { when } v_{i} \text { points to } v_{j} \text { by an arc } \\ 0 & \text { otherwise, }\end{cases}
$$




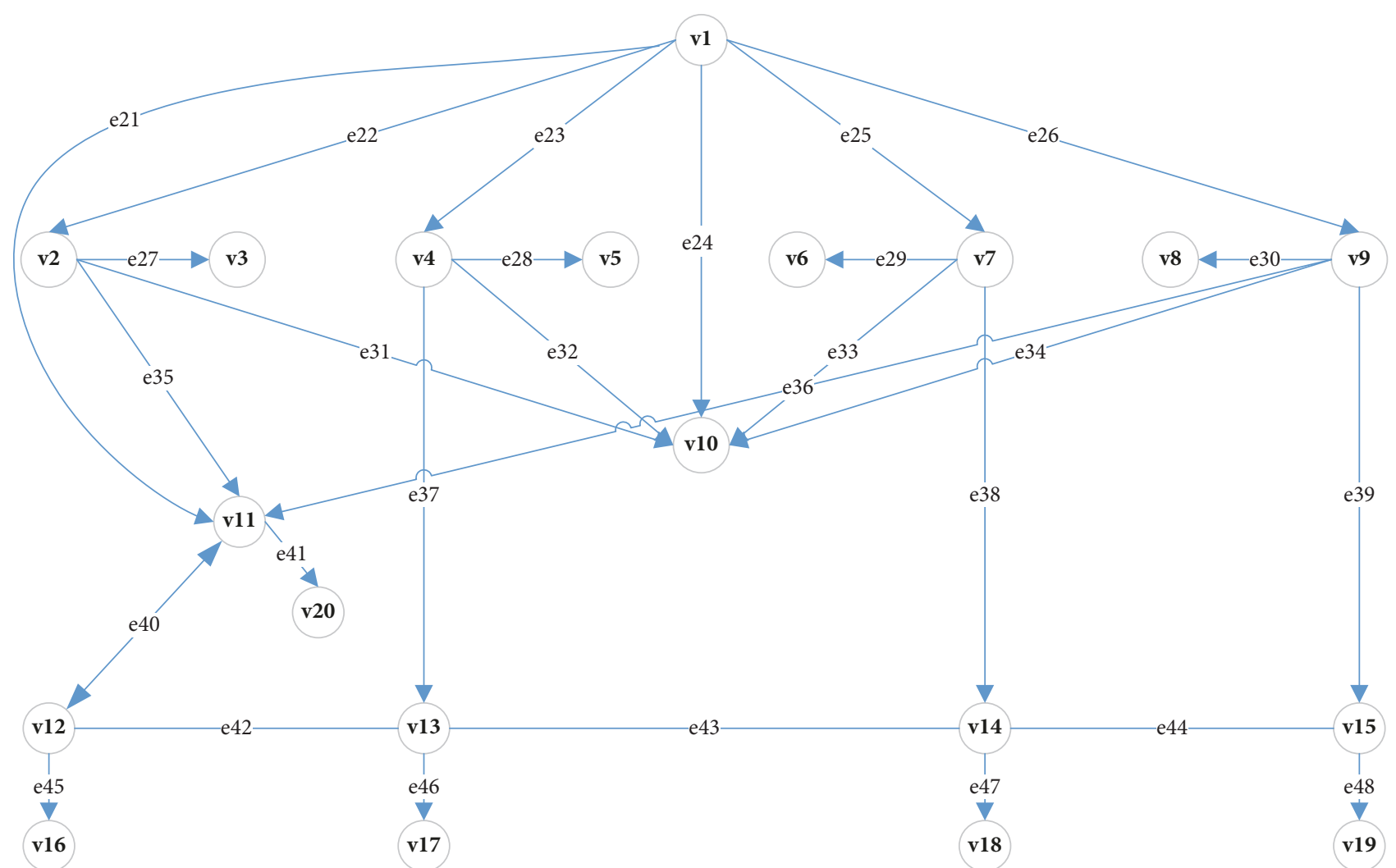

FIGURE 2: Network nodes graph.

where superscript 1 represents that the path length of the adjacent matrix is 1 , it also represents the first iteration matrix in the calculation. Variables $i$ and $j$ stand for the row and column of the matrix, respectively.

The terminal matrix $R=\left[r_{j k}\right]$ shows the terminal point of each arc in the network nodes diagram $N$

$$
\begin{aligned}
& r_{j k} \\
& \quad= \begin{cases}v_{k} & \text { when } v_{j} \text { and } v_{k} \text { are connected by an arc } \\
0 & \text { otherwise or } i=j .\end{cases} \\
& \left\{a^{1}{ }_{i j} ※ \text { " is defined as an operation with the algorithm of } a^{2}{ }_{i k} \mid j=1,2, \ldots, n\right\}:
\end{aligned}
$$

$$
a_{i j}^{1} \circledast r_{j k} \begin{cases}v_{i} v_{j} v_{k} & \text { when } a^{1}{ }_{i j}=v_{i} v_{j}, r_{j k}=v_{k} \text { and } v_{i}, v_{j}, v_{k} \text { are different } \\ 0 & \text { when } a^{1}{ }_{i j}=0 \text { or } r_{j k}=0 \text { or there is the same pair among } v_{i}, v_{j}, v_{k} \text { at least. }\end{cases}
$$

In the calculation process, one element of $A_{n}$ is made up of one character array. In order to save memory and facilitate further calculation, any character array in $A_{n}$ is converted into a character string by neglecting its zero and linking its nonzero character with "+." For example, $A 2(1,2)=$ $\{0,0,0$, "v1v10v2", "v6", $\ldots, 0\}$ is simplified to $A 2(1,2)=$ \{“v1v10v2 + v6"\}.

The meaning of adjacent matrix $A$ indicates that $A_{1}$ contains all minimal paths with length of 1 from $v_{i}$ to $v_{k}$. The same can be obtained that length of $A_{2}$ is 2 . The length of longest path of network node diagram $N$ may be $n-1$, and $A_{t}(t=1,2,3, \ldots, n-1)$ includes all sets of paths from any arbitrary node to another.

Compared with the Depth-First Search, the adjacent matrix algorithm has the characteristics of clear concept, simple rules, and so on, then it is faster in the low rank matrixes and occupies less memory. In consideration of the fact that network node diagram converted from EMA power system has characteristics of simple structure and less nodes, adjacent matrix algorithm can be used to obtain the minimal path set of network node diagram. This method is more practical than others.

Figure 4 is the flow chart of adjacent matrix algorithm, in which $i$ and $k$ stand for a row vector and a column vector, respectively, $j$ stands for the number of participating nodes in each operation step, and $m$ is length of path. According to the practical application, only the paths with specific length are calculated from the source node to specific nodes. 


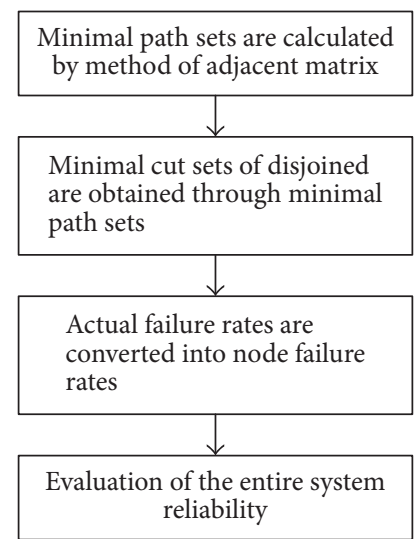

FIGURE 3: Flow chart of reliability assessment.

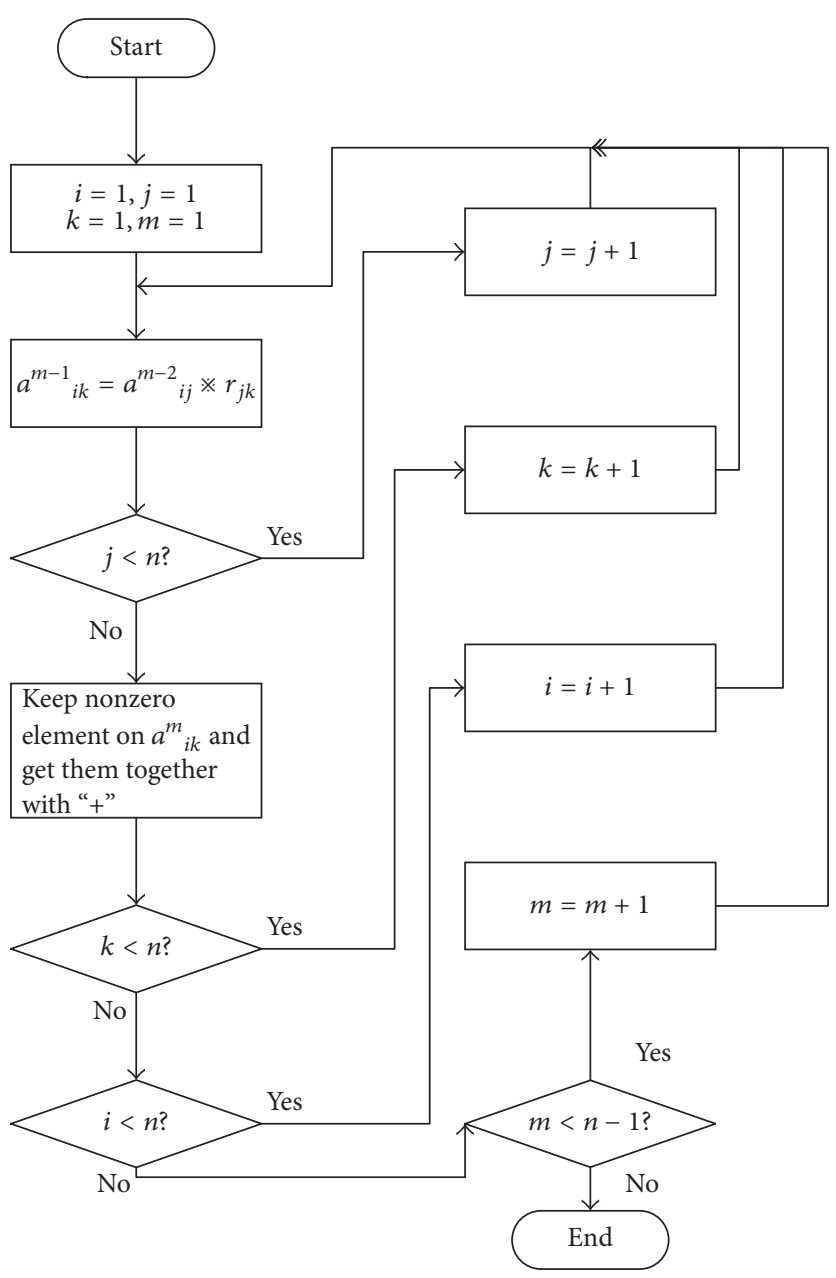

FIGURE 4: Flow chart of obtaining minimal path sets by adjacent matrix.

4.2. Calculation of Disjointed Minimal Cut Sets. In the same network node diagram, minimal cut sets and minimal path sets are interchangeable. To calculate the probability of fault occurrence, the minimal path sets are converted into the minimal cut sets. If a node fails, this means that the assigned node must be out of power. Then component fault represented by this node is the first-order minimal cut set of this network node diagram. Except the node fault represented by first-order minimal cut sets, the other paths include the same two components or any of them, and the fault of the two components is second-order minimal cut sets. Similarly, minimal cut sets from source node to assigned node can be calculated, including first-, second-, third-, high-order minimal cut sets, corresponding to single fault, double faults, 


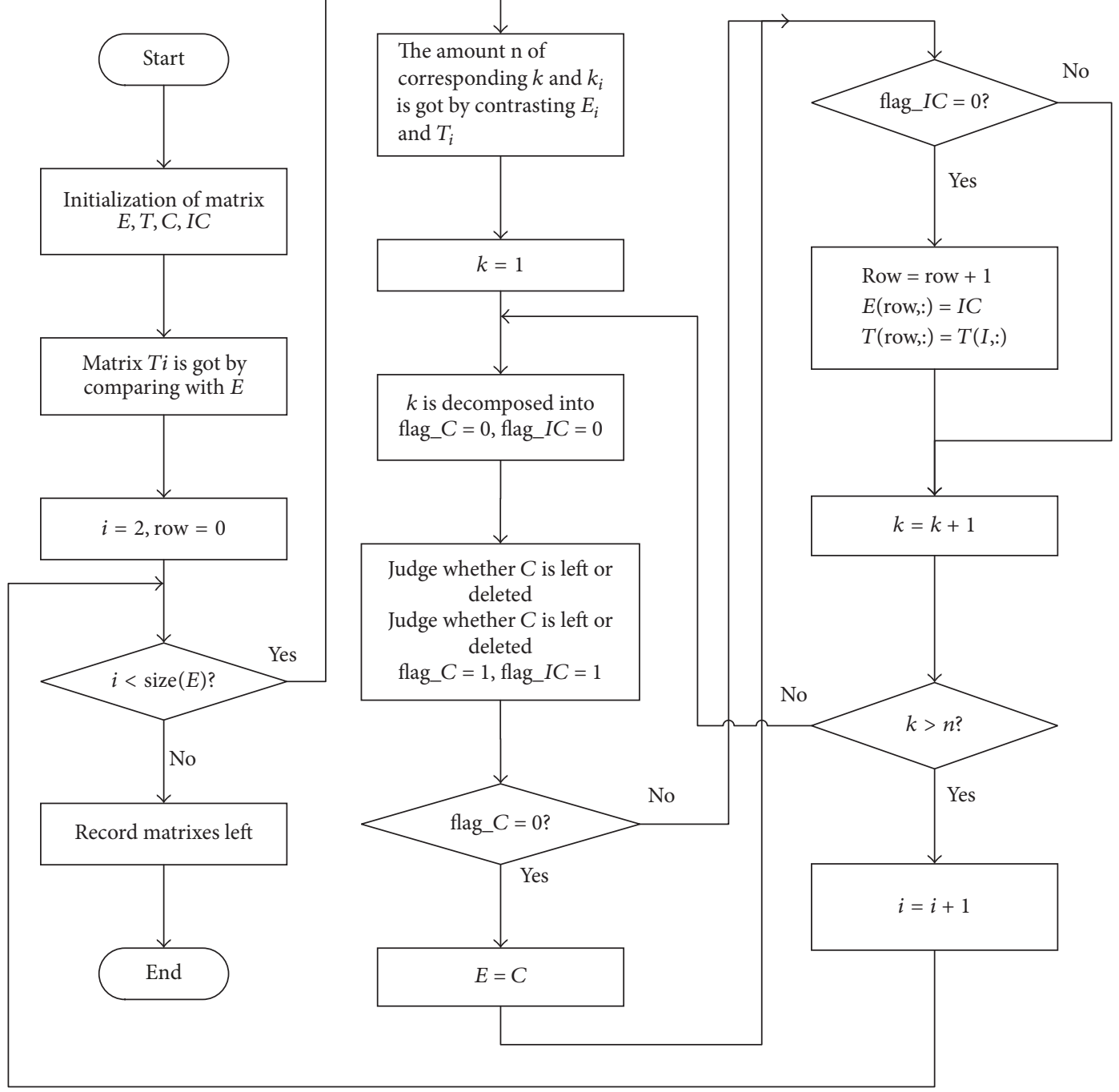

FIGURE 5: Flow chart of Delete-Leave Method.

triple faults, multiple faults, and so on. As matter of fact, loworder faults are the main cause of influence on reliability, so quadruple and blew faults are considered in analysis. The conversion method is very mature and no more detail here [11].

Assuming a minimal cut set is $S_{n}$, and system event is $S=$ $S_{1}+S_{2}+\cdots+S_{n}$. And analysis of $S_{1}, S_{2}, \ldots, S_{n}$ shows that there is overlap among different minimal cut sets. Then probability of $S$ is expressed by

$$
\begin{aligned}
P(S)= & \sum_{i}^{n} p\left(S_{i}\right)-\sum_{1<i<j<n}^{n} p\left(S_{i} S_{j}\right)+\sum_{1<i<j<k<n}^{n} p\left(S_{i} S_{j} S_{k}\right) \\
& -\cdots+(-1)^{n-1} \sum_{1<i<j<k<\cdots<n}^{n} p\left(S_{1} S_{2} \cdots S_{n}\right) .
\end{aligned}
$$

The number of items in (4) is up to $2^{n}-1$, and the amount of calculation increases exponentially when $n$ is larger. In order to improve the computational efficiency, it is necessary to deal with minimal cut sets by disjointing. The Method of Delete-Leave proposed by Aggarwal [12] is adopted, of which the basic idea is to the disjoint minimal cut sets by adding variables.

The flow chart of Method of Delete-Leave is shown in Figure 5, then the following is variable description and specific implementation steps:

(1) Define $n$-dimensional vector $E_{i}\left(x_{1}, x_{2}, \ldots, x_{n}\right)$ for each minimal cut set, and $x_{i}$ can be valued at 0 or 1 . $x_{i}=1$ means that the minimal cut set includes node or arc; otherwise $x_{i}=0$.

(2) The auxiliary matrix $T_{i}\left(t_{1}, t_{2}, \ldots, t_{n}\right)$ is defined and the following condition is satisfied:

$$
t_{i}=\left\{\begin{array}{l}
1 \quad \text { when sum of components } e_{i} \text { is more than } 1 \text { from } E_{1} \text { to } E_{i} \\
0 \quad \text { when sum of components } e_{i} \text { is more than } 0 \text { from } E_{1} \text { to } E_{i} .
\end{array}\right.
$$


(3) Suppose $E_{1}$ is the first disjoint minimal cut set. When $i>2$, find positions at which elements in $T_{i}$ are 1 and $E_{i}$ are 0 , and these positions are recorded in sequence as $k_{1}, k_{2}, \ldots, k_{r}$ according to the size of order.

(4) Decompose $E_{i}$ into $E_{i}\left(k_{1}\right)$ and $E_{i}\left(\overline{k_{1}}\right)$ in sequence according to $k_{i}(i=1,2,3, \ldots, r)$; 0 in this position of $k_{i}$ in $E_{i}$ is replaced with 1 or -1 , respectively.

(5) Comparing $E_{i}\left(k_{1}\right)$ with $E_{j}(j<i), E_{i}\left(k_{1}\right)$ is incompatible with $E_{j}$ on condition that position where elements in $E_{i}\left(k_{1}\right)$ are -1 corresponding to relevant position of $E_{j}$ is 1 and the number of 1 is equal to the rows $j . E_{i}\left(k_{1}\right)$ is left as disjoint minimal cut set and $E_{i}\left(\overline{k_{1}}\right)$ is treated in the same way.

(6) Contrasting $T_{i-1}$ with $E_{i}\left(k_{1}\right)$ which is not left, it has thought that $E_{i}\left(k_{1}\right)$ is absorbed by $E_{j}(j<i)$ on condition that position which in $T_{i-1}$ is not 0 corresponding to relevant position of $E_{i}\left(k_{1}\right)$ is not 0 . There is an overlap between $E_{i}\left(k_{1}\right)$ and anyone in $E_{j}$, so $E_{i}\left(k_{1}\right)$ is deleted and $E_{i}\left(\overline{k_{1}}\right)$ is treated in the same way.

(7) $E_{i}\left(k_{1}\right)$ is decomposed into $E_{i}\left(k_{1}\right)\left(k_{2}\right)$ and $E_{i}\left(k_{1}\right)\left(\overline{k_{2}}\right)$ continually if $E_{i}\left(k_{1}\right)$ is neither left nor deleted. Replace 0 in position of $k_{2}$ with 1 or -1 , and steps (4), (5), and (6) are repeated until a decision is made between deleting and leaving. The final results obtained are all disjoint minimal cut sets, and the probability of event $S$ could be obtained by

$$
P(S)=\sum_{1}^{n} P\left(S_{i}\right)
$$

4.3. Reliability Data Conversion. Assume that all the components of MEA power system are irreparable. The irreparability refers to that the element cannot be repaired in time rather than technically impossible when a fault occurs. And quantitative indexes include failure rate $\lambda$, life time $t$, and degree of reliability $R_{s}$. Suppose that $\lambda$ is constant, and its life time obeys exponential distribution, then $R_{s}(t)=e^{-\lambda t}$.

Every component has its own failure rate $\lambda$, and failure rate of each node differs from factory test data of a single component. Components interact with each other when a fault occurs and the protection devices make different response under fault conditions. Therefore, it is necessary to convert factory test data or so called actual failure rate into node failure rate in reliability calculation.

Based on the following three methods, the actual failure rate is converted to the node failure rate of network node diagram. If the components are all qualified when they leaved factory, their service lifetime should obey the exponential distribution. That is, the failure rate of component is a constant. According to the actual experience, assuming that all faults occur on components and wire connections are in good condition, then there is no rosin joint on connection points.

The failure rate of arc representing components connected in series can be converted according to (7) on the network node diagram.

$$
\lambda_{e}=\sum_{i=1}^{n} \lambda_{i}
$$

$\lambda_{i}$ stands for failure rate of each component in series; $n$ stands for the number of serial components in this arc; $\lambda_{e}$ stands for equivalent failure rate of the arc after conversion.

When a node is connected with multiple arcs in network node diagram, a failure of arc which is not eliminated in time could lead to a diffusive failure even to cause cascading outages to make normal nodes out of service. The failure of the node, due to quitting the operation caused by diffusive failure, is also converted to node failure rate. In the actual conversion only fault of first-order protective devices is considered.

The calculated failure rate of a single node is composed of three parts. The first part is the actual failure rate $\lambda_{b}$. The second part is the diffusive failure which is caused by the faults of adjacent node or arc without no protection devices. The diffusive failure rate is denoted as $\lambda_{e k}$ and the number of nonprotective devices the fault passing through is denoted as $w$. The third part is the diffusive failure which is caused by the faults of adjacent node or arc when protective devices refuse operation. The number of protective devices which the faults pass by is denoted as $m$ and the number of protective devices or buffering is denoted as $u$ before the fault is isolated. Reliability degree of a node can calculated by

$$
\lambda_{e b}=\lambda_{b}+\sum_{1}^{w} \lambda_{e k}+\sum_{1}^{m}\left(P_{f i} \sum_{1}^{u} \lambda_{e k}\right) .
$$

$P_{f i}$ is the fault transmissibility of a protective device in (8), which is equal to the fact that the number of refuse operations is divided by the number of the protective actions.

After disjointing of minimal cut sets and conversion of actual failure rate, reliability of assigned nodes for power supply is calculated by (6).

\section{Case Study}

5.1. Calculation of Reliability Data. Above methods are used and A380 is taken as an example to evaluate the reliability. Three typical Nodes v6, v18, and v20 are chosen to be comparatively analyzed on the basis of Figures 1 and 2. The reliability of the entire system is evaluated by evaluating reliability of assigned nodes. Node v6 stands for an AC load directly supplied by the AC bus bar (AC3). Node v18 stands for a DC load directly supplied by the DC bus bar (DC3). Node v20 stands for the key loads to maintain flying condition supplied by the AC bus bars for equipment. The $\mathrm{AC}$ bus bar for key equipment is supplied by dual channels $\mathrm{ACl}$ and $\mathrm{AC} 4$. On emergency, the RAT is unfolded to provide emergency supply power and DC power from batteries is inverted to AC as supplementary by static convertors, so as to ensure the uninterrupted power supply of key equipment.

The minimal path sets and minimal cut sets are calculated by above methods on the basis of network node diagram shown in Figure 2, and the results are shown in Tables 1 and 2. In application, low-order faults are main reason that affects reliability, so the minimal cut sets are calculated to the fourth order. Finally, the minimal cut sets are disjointed by using Method of Delete-Leave. 
TABLE 1: Minimal path sets from source to different nodes.

\begin{tabular}{|c|c|c|c|}
\hline Length of path & Node 6 & Node 18 & Node 20 \\
\hline 2 & v1v7v6 & & v1v11v20 \\
\hline \multirow{2}{*}{3} & v1v10v7v6 & v1v7v14v18 & v1v2v11v20 \\
\hline & & & v1v9v11v20 \\
\hline \multirow{3}{*}{4} & v1v2v10v7v6 & v1v10v7v14v18 & v1v10v2v11v20 \\
\hline & v1v4v10v7v6 & v1v4v13v14v18 & v1v10v9v11v20 \\
\hline & v1v9v10v7v6 & v1v9v15v14v18 & \\
\hline \multirow{7}{*}{5} & & v1v2v10v7v14v18 & v1v4v10v2v11v20 \\
\hline & & v1v4v10v7v14v18 & v1v7v10v2v11v20 \\
\hline & & v1v9v10v7v14v18 & v1v9v10v2v11v20 \\
\hline & & v1v10v4v13v14v18 & v1v2v10v9v11v20 \\
\hline & & v1v11v12v13v14v18 & v1v4v10v9v11v20 \\
\hline & & v1v10v9v15v14v18 & v1v7v10v9v11v20 \\
\hline & & & v1v4v13v12v11v20 \\
\hline \multirow{8}{*}{6} & & v1v2v10v4v13v14v18 & v1v10v4v13v12v11v20 \\
\hline & & v1v7v10v4v13v14v18 & v1v7v14v13v12v11v20 \\
\hline & & v1v9v10v4v13v14v18 & \\
\hline & & v1v2v11v12v13v14v18 & \\
\hline & & v1v9v11v12v13v14v18 & \\
\hline & & v1v2v10v9v15v14v18 & \\
\hline & & v1v4v10v9v15v14v18 & \\
\hline & & v1v7v10v9v15v14v18 & \\
\hline \multirow{5}{*}{7} & & v1v10v2v11v12v13v14v18 & v1v2v10v4v13v12v11v20 \\
\hline & & v1v10v9v11v12v13v14v18 & v1v7v10v4v13v12v11v20 \\
\hline & & & v1v9v10v4v13v12v11v20 \\
\hline & & & v1v10v7v14v13v12v11v20 \\
\hline & & & v1v9v15v14v13v12v11v20 \\
\hline \multirow{6}{*}{8} & & v1v4v10v2v11v12v13v14v18 & v1v2v10v7v14v13v12v11v20 \\
\hline & & v1v7v10v2v11v12v13v14v18 & v1v4v10v7v14v13v12v11v20 \\
\hline & & v1v9v10v2v11v12v13v14v18 & v1v9v10v7v14v13v12v11v20 \\
\hline & & v1v2v10v9v11v12v13v14v18 & v1v10v9v15v14v13v12v11v20 \\
\hline & & v1v4v10v9v11v12v13v14v18 & \\
\hline & & v1v7v10v9v11v12v13v14v18 & \\
\hline \multirow{3}{*}{9} & & & v1v2v10v9v15v14v13v12v11v20 \\
\hline & & & $\mathrm{v} 1 \mathrm{v} 4 \mathrm{v} 10 \mathrm{v} 9 \mathrm{v} 15 \mathrm{v} 14 \mathrm{v} 13 \mathrm{v} 12 \mathrm{v} 11 \mathrm{v} 20$ \\
\hline & & & v1v7v10v9v15v14v13v12v11v20 \\
\hline
\end{tabular}

Table 3 shows actual failure rate and diffusive failure rate of components of EMA power system. Table 4 shows conversion equation and specific reliability degree of nodes or arcs.

5.2. Reliability Evaluation and Analysis. The reliability evaluation results on key loads v20, AC loads v18, and DC loads v6 are shown in Figure 6.

Define the length of an arc as electrical distance 1. Theoretically, the longer the electrical distance from the loads to the source is, the lower the degree of reliability is. Based on Figures 1 and 6, it can be found that Node 20 has longest electrical distance from the source; however, its degree of reliability in a short time is higher than Node 18 and Node 6. It shows that the way to increase the reliability by adding redundant lines in actual operation can only achieve better results in a period of time. However, as the running time increases, decline speed of reliability for Node 20 is going faster and faster. It can be seen that redundant lines cannot solve problems fundamentally, and key of problem lies in the length of electrical distance from loads to the source.

Reliability of key loads is less than that of general loads at about 2300 hours from Figure 6, and this may be the reason that C-check of many civil aircraft is about 2000 hours.

From the physical point of view, Node v20 stands for key load which is supplied by AC bus bar for key equipment v1l. Four power supply channels are equipped to ensure the uninterrupted power supply of Node v1l. Power is supplied from the right and left of generators through AC bus bars (AC1 and AC4). When one side of generators breaks down, 
TABLE 2: Minimal cut sets causing nodes permanent loss of electricity.

\begin{tabular}{|c|c|c|c|}
\hline & Node 6 & Node 18 & Node 20 \\
\hline (1) & v6 & v14 & v11 \\
\hline (2) & e29 & v18 & v20 \\
\hline (3) & v7 & e 48 & $\mathrm{e} 42$ \\
\hline (4) & e25v10 & v7v9v13 & v2v9v12e21 \\
\hline (5) & e25e33 & v7v9e44 & v2v9v13e21 \\
\hline (6) & & v7v13v15 & v2v9e41e21 \\
\hline (7) & & v7v13e39 & v2v9e $43 e 21$ \\
\hline (8) & & v7v13e45 & v2v12e21e36 \\
\hline (9) & & v7v15e44 & v2v13e21e36 \\
\hline (10) & & v7e39e44 & v2v21e36e41 \\
\hline (11) & & v7e44e45 & v2e21e $36 \mathrm{e} 43$ \\
\hline (12) & & v9v13e38 & v9v12e21e35 \\
\hline (13) & & v9e38e44 & v9v13e21e35 \\
\hline (14) & & v13v15e38 & v9e21e35e41 \\
\hline (15) & & v13e38e39 & v9e21e35e43 \\
\hline (16) & & v13e $38 \mathrm{e} 45$ & v12e $21 \mathrm{e} 35 \mathrm{e} 36$ \\
\hline (17) & & v15e38e44 & v13e21e35e36 \\
\hline (18) & & e38e39e44 & e21e35e36e41 \\
\hline (19) & & e $38 \mathrm{e} 44 \mathrm{e} 45$ & $\mathrm{e} 21 \mathrm{e} 35 \mathrm{e} 36 \mathrm{e} 43$ \\
\hline
\end{tabular}

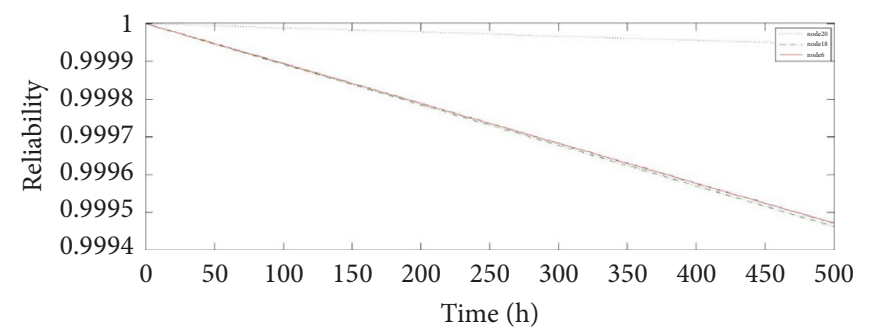

(a) Reliability of different nodes in 500 hours

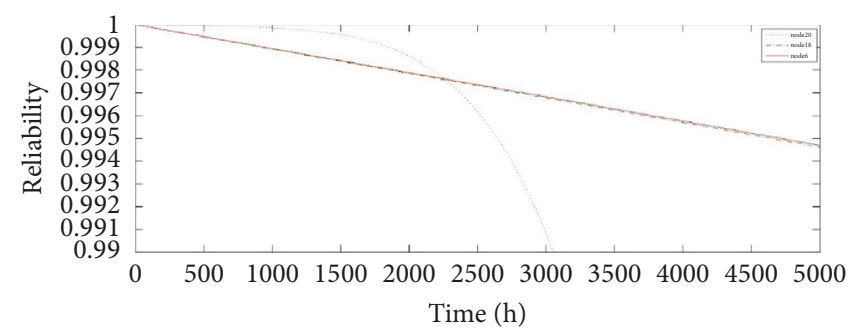

(b) Reliability of different nodes in 5000 hours

FIGURE 6: Reliability of different nodes.

TABLE 3: Row reliability data of a portion of components.

\begin{tabular}{lcc}
\hline Components & Failure rate $/ \mathrm{h}^{-1}$ & Diffusive failure rate $/ \mathrm{h}^{-1}$ \\
\hline Contactor & $1.3333 * 10^{-5}$ & $\begin{array}{c}0.5 \% \\
\text { (malfunction rate) }\end{array}$ \\
Generator & $5.5556 * 10^{-5}$ & $8.3333 * 10^{-6}$ \\
Inverter & $9.0909 * 10^{-5}$ & $1.3636 * 10^{-5}$ \\
Battery & $1.8300 * 10^{-6}$ & $2.7450 * 10^{-7}$ \\
TRU & $5.8824 * 10^{-5}$ & $8.8235 * 10^{-6}$ \\
Bus & $5.0000 * 10^{-7}$ & $5.0000 * 10^{-7}$ \\
Diode & $5.7143 * 10^{-6}$ & $8.5714 * 10^{-7}$ \\
Feeder & $3.4483 * 10^{-6}$ & $1.5517 * 10^{-6}$ \\
\hline
\end{tabular}

the other side would ensure the uninterrupted power supply for vll. The DC energy is converted to supply power for vll through static converter when necessary. On the final emergency, the MEA unfolds RAT to supply power for vll directly.
The DC loads include environmental control system and avionic system, of which reliability demand is relatively high. DC bus bars have relative reliability, because they operate in parallel which is equivalent to three power supply channels, and the batteries are plugged into DC bus bars. AC loads include heating, lighting, and kitchen loads. Compared with other loads, their reliability demands are low and can be met by fed from AC bus bars.

\section{Conclusion}

In view of the structural characteristics of MEA power system, taking A380 as an example, an equivalent method for electrical structure and a reliability evaluation are proposed. The disjointed minimal cut sets are calculated by minimal path sets of relevant events and Method of Delete-Leave. The reliability of representative node is obtained by converting failure rate, which could describe the reliability of the entire system. 
TABLE 4: Conversion reliability of relevant nodes (arcs).

\begin{tabular}{|c|c|c|}
\hline Nodes or arcs & Formula & Reliability \\
\hline 2 & $P_{\mathrm{Bus}}+0.5 \% *\left(P_{\mathrm{Generator}}+P_{\mathrm{Feeder}} * 3\right)$ & $5.6494 * 10^{-7} / \mathrm{h}^{-1}$ \\
\hline 6 & $P_{\mathrm{Bus}}+0.5 \% * P_{\mathrm{Feeder}}$ & $5.0776 * 10^{-7} / \mathrm{h}^{-1}$ \\
\hline 7 & $P_{\text {Bus }}+0.5 \% *\left(P_{\text {Generator }}+P_{\text {Feeder }} * 2+P_{\mathrm{TRU}}\right)$ & $6.0130 * 10^{-7} / \mathrm{h}^{-1}$ \\
\hline 9 & $P_{\text {Bus }}+0.5 \% *\left(P_{\text {Generator }}+P_{\text {Feeder }} * 3+P_{\text {TRU }}\right)$ & $6.0906 * 10^{-7} / \mathrm{h}^{-1}$ \\
\hline 10 & $P_{\text {Bus }}+0.5 \% *\left(P_{\text {Feeder }} * 4+P_{\text {Generator }}\right)$ & $5.7270 * 10^{-7} / \mathrm{h}^{-1}$ \\
\hline 11 & $P_{\mathrm{Bus}}+0.5 \% *\left(P_{\text {Generator }}+P_{\text {Feeder }} * 2+P_{\text {Inverter }}+P_{\mathrm{TRU}} * 2\right)$ & $5.1360 * 10^{-7} / \mathrm{h}^{-1}$ \\
\hline 12 & $P_{\mathrm{Bus}}+0.5 \% *\left(P_{\text {Inverter }}+P_{\text {Feeder }} * 2+P_{\mathrm{TRU}}\right)$ & $6.2781 * 10^{-7} / \mathrm{h}^{-1}$ \\
\hline 13 & $P_{\mathrm{Bus}}+0.5 \% *\left(P_{\mathrm{Feeder}} * 3+P_{\mathrm{TRU}}\right)$ & $5.6739 * 10^{-7} / \mathrm{h}^{-1}$ \\
\hline 14 & $P_{\mathrm{Bus}}+0.5 \% *\left(P_{\mathrm{Feeder}} * 3+P_{\mathrm{TRU}}\right)$ & $5.6739 * 10^{-7} / \mathrm{h}^{-1}$ \\
\hline 15 & $P_{\mathrm{Bus}}+0.5 \% *\left(P_{\mathrm{Feeder}} * 2+P_{\mathrm{TRU}}\right)$ & $5.5963 * 10^{-7} / \mathrm{h}^{-1}$ \\
\hline 18 & $P_{\text {Bus }}+0.5 \% * P_{\text {Feeder }}$ & $5.0776 * 10^{-7} / \mathrm{h}^{-1}$ \\
\hline 20 & $P_{\mathrm{Bus}}+0.5 \% * P_{\mathrm{TRU}}$ & $5.4412 * 10^{-7} / \mathrm{h}^{-1}$ \\
\hline 21 & $P_{\text {Feeder }} * P_{\text {Generator }} * P_{\text {Contactor }}$ & $2.5542 * 10^{-15} / \mathrm{h}^{-3}$ \\
\hline 25 & $P_{\mathrm{Feeder}} * P_{\mathrm{Generator}} * P_{\text {Contactor }}$ & $6.1300 * 10^{-16} / \mathrm{h}^{-3}$ \\
\hline 29 & $P_{\text {Contactor }} * P_{\text {Contactor }} * P_{\mathrm{TRU}} * P_{\text {Feeder }}$ & $3.6059 * 10^{-20} / \mathrm{h}^{-4}$ \\
\hline 33 & $P_{\text {Contactor }} * P_{\text {Contactor }} * P_{\text {Feeder }}$ & $6.1300 * 10^{-16} / \mathrm{h}^{-3}$ \\
\hline 35 & $P_{\text {Contactor }} * P_{\text {Contactor }} * P_{\text {Feeder }}$ & $6.1300 * 10^{-16} / \mathrm{h}^{-3}$ \\
\hline 36 & $P_{\text {Contactor }} * P_{\text {Contactor }} * P_{\text {Feeder }}$ & $6.1300 * 10^{-16} / \mathrm{h}^{-3}$ \\
\hline 38 & $P_{\text {Contactor }} * P_{\text {Contactor }} * P_{\text {Feeder }} * P_{\text {TRU }}$ & $3.6059 * 10^{-20} / \mathrm{h}^{-4}$ \\
\hline 39 & $P_{\text {Contactor }} * P_{\text {Contactor }} * P_{\text {Feeder }} * P_{\mathrm{TRU}}$ & $3.6059 * 10^{-20} / \mathrm{h}^{-4}$ \\
\hline 41 & $P_{\text {Contactor }} * P_{\text {Contactor }} * P_{\text {Inverter }} * P_{\text {Feeder }}$ & $5.5727 * 10^{-20} / \mathrm{h}^{-4}$ \\
\hline 42 & $P_{\text {Contactor }} * P_{\text {Contactor }} * P_{\mathrm{TRU}} * P_{\mathrm{Feeder}}$ & $3.6059 * 10^{-20} / \mathrm{h}^{-4}$ \\
\hline 43 & $P_{\text {Contactor }} * P_{\text {Contactor }} * P_{\text {Feeder }}$ & $6.1300 * 10^{-16} / \mathrm{h}^{-3}$ \\
\hline 44 & $P_{\text {Contactor }} * P_{\text {Contactor }} * P_{\text {Feeder }}$ & $6.1300 * 10^{-16} / \mathrm{h}^{-3}$ \\
\hline 45 & $P_{\text {Contactor }} * P_{\text {Contactor }} * P_{\text {Feeder }}$ & $6.1300 * 10^{-16} / \mathrm{h}^{-3}$ \\
\hline 48 & $P_{\text {Contactor }} * P_{\text {Contactor }} * P_{\mathrm{TRU}} * P_{\text {Feeder }}$ & $3.6059 * 10^{-20} / \mathrm{h}^{-4}$ \\
\hline
\end{tabular}

The proposed equivalent method and reliability evaluation method could analyze the reliability of the MEA power system conveniently and effectively. It is only for a period of time that the method of adding redundant lines to increase reliability can make up for the lack of reliability of the remote components. The method proposed in this article can provide support for formulation of maintenance scheme about MEA power system. Meanwhile, it has a reference value for optimization and improvement of the later power structure.

\section{Conflicts of Interest}

The authors declare that they have no conflicts of interest.

\section{Acknowledgments}

The authors would like to thank financial support from National Natural Science Foundation of China (U1533126).

\section{References}

[1] R. Qi, H. Lin, and S.-y. Zhou, "Study on key techniques for electrical system of more electric aircraft," Aeronautical Computer Technique, vol. 34, no. 1, pp. 97-101, 2004.
[2] I. Moir and A. Seabridge, Aircraft Systems: Mechanical, Electrical, and Avionics Subsystems Integration, 3rd edition, 2008.

[3] Z. Suying, Study on Electrical System of More Electric Aircraft, Northwestern Polytechnical University, 2008.

[4] X. Bian, Y. Zhou, and Q. Ma, “Topology of power system reliability in more electric aircraft," Beijing Hangkong Hangtian Daxue Xuebao/Journal of Beijing University of Aeronautics and Astronautics, vol. 34, no. 10, pp. 1210-1213, 2008.

[5] M. A. Maldonado, N. M. Shah, K. J. Cleek, and G. J. Korba, "Power management and distribution system for a moreelectric aircraft (MADMEL): Program status," IEEE Aerospace and Electronic Systems Magazine, vol. 14, no. 12, pp. 3-8, 1999.

[6] R. Billinton and P. Wang, "Distribution system reliability cost/worth analysis using analytical and sequential simulation techniques," IEEE Transactions on Power Systems, vol. 13, no. 4, pp. 1245-1250, 1998.

[7] Y. M. Atwa and E. F. El-Saadany, "Reliability evaluation for distribution system with renewable distributed generation during islanded mode of operation," IEEE Transactions on Power Systems, vol. 24, no. 2, pp. 572-581, 2009.

[8] S. Ramabhotla, S. Bayne, and M. Giesselmann, "Reliability optimization using fault tree analysis in the grid connected mode of microgrid," in Proceedings of the 8th Annual IEEE Green Technologies Conference, GreenTech 2016, pp. 136-141, Kansas City, MO, USA, April 2016. 
[9] Y. Dun, W. Shoufang, and L. Xia, Foreign Aircraft Power Supply System Manual, China Aeroinfo Center, Beijing, China, 1997.

[10] Q. Haihong and Y. Yangguang, Power System for More Electric Aircraft, Beihang University Press, 2016.

[11] G. Yongji, Principle of Reliability Engineering, Tsinghua University press, 2002.

[12] S. Rai and K. K. Aggarwal, "An Efficient Method for Reliability Evaluation of a General Network," IEEE Transactions on Reliability, vol. R-27, no. 3, pp. 206-211, 1978. 


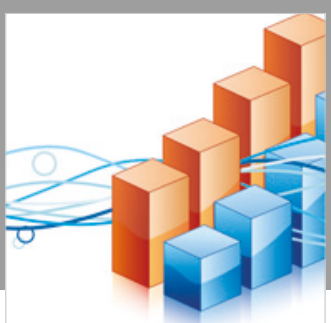

Advances in

Operations Research

\section{-n-m}
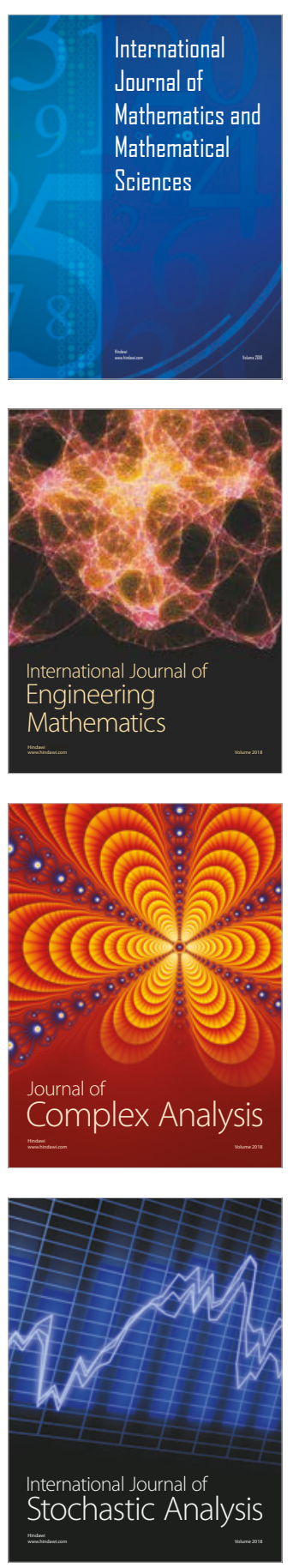
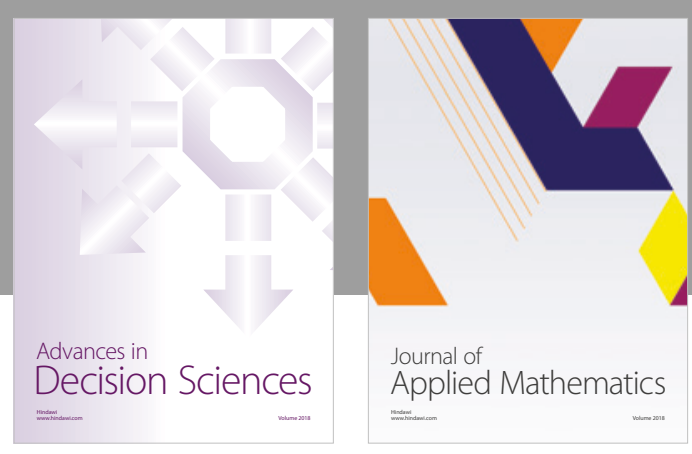

Journal of

Applied Mathematics
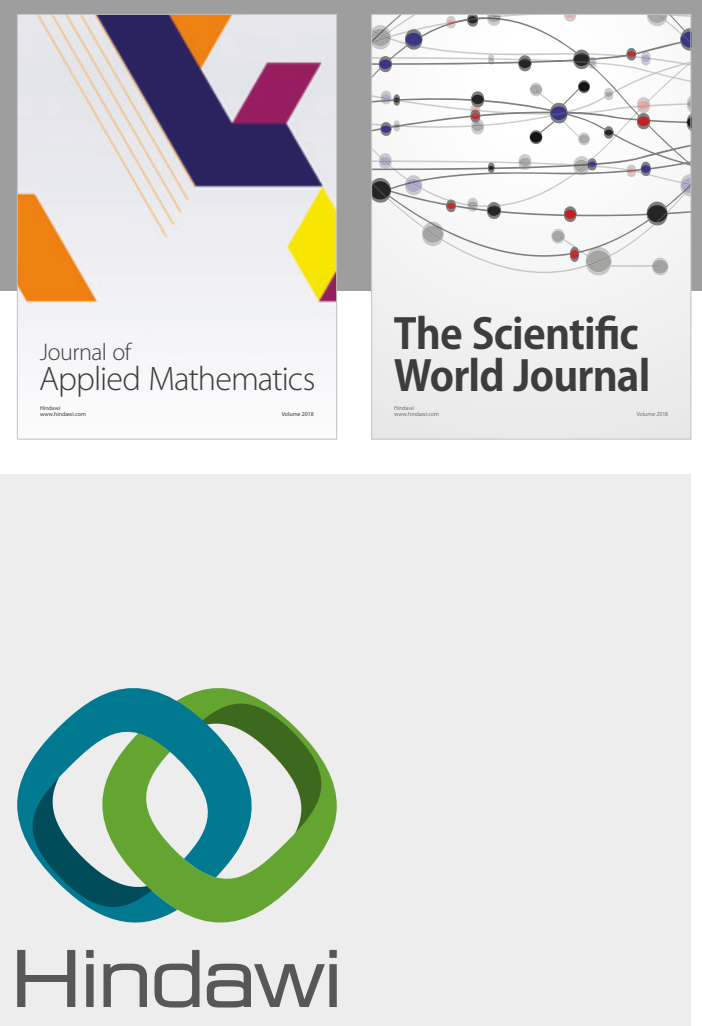

Submit your manuscripts at

www.hindawi.com

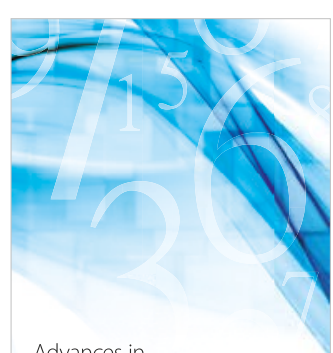

Advances in
Numerical Analysis
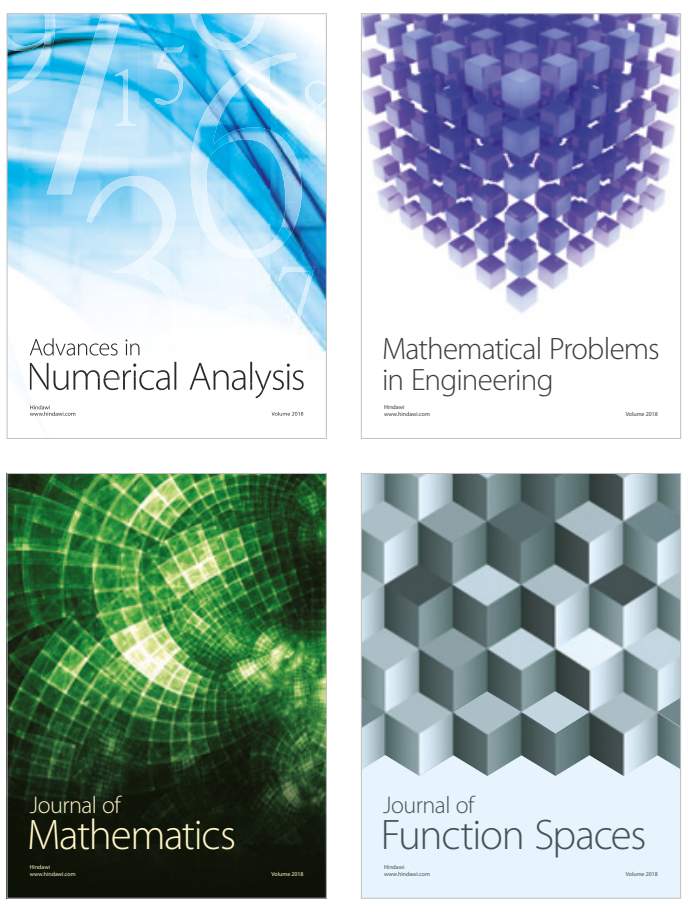

Mathematical Problems in Engineering

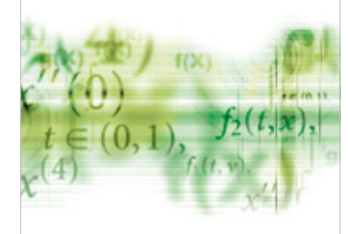

International Journal of

Differential Equations

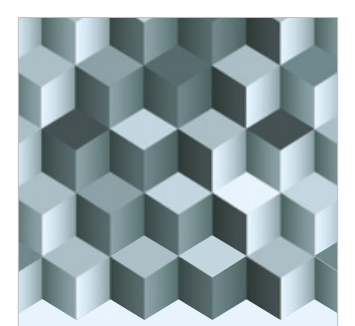

Journal of

Function Spaces

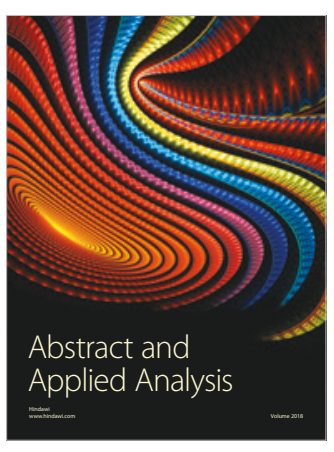

The Scientific

World Journal

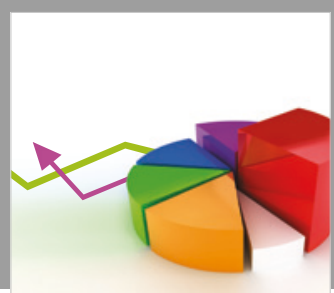

Journal of

Probability and Statistics
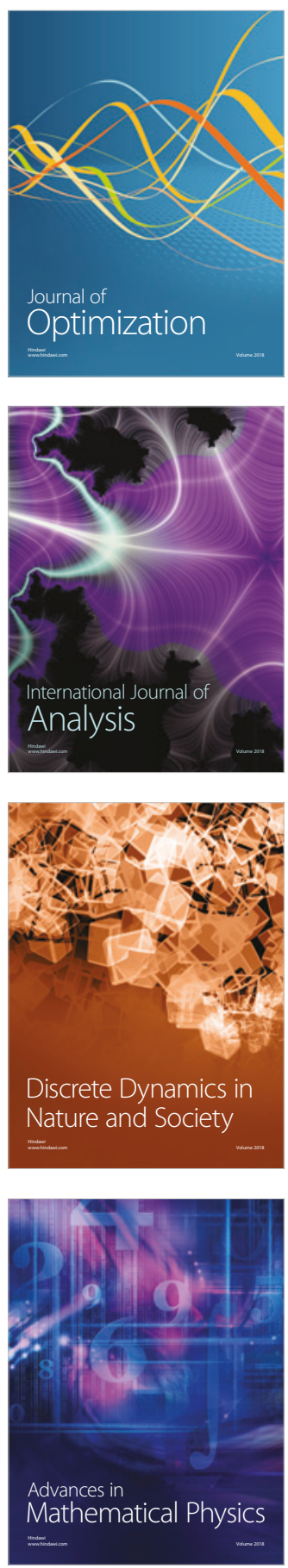